\title{
Percolative behavior of an anisotropic two-dimensional network: Growth of tellurium onto an oriented polymer film
}

\author{
T. Hoffmann \\ Fochbereich Elektrotechnik, Lehrstuhl für Materiolien in der Elektrotechnik, Fuhlrottstr. 10, 42097 Wuppertal, Germany \\ J. Martínez-Salazar \\ Instituto de Estructura de la Materia, Consejo Superior de Investigaciones Científicas, Serrano 119, 28006 Madrid, Spain \\ P. Herrero \\ Instituto de Ciencia de Materiales, Consejo Superior de Investigaciones Científicas, Serrano 113, 28006 Madrid, Spain \\ J. Petermann \\ Fachbereich Chemietechnik, Lehrstuhl für Werkstoffkunde, Universität Dormund, Postfach 500500, 44221 Dortmund, Germany
}

(Received 31 January 1996; revised manuscript received 26 August 1996)

\begin{abstract}
In situ transport measurements during the growth of a thin layer of thermally evaporated tellurium onto an oriented polymer film are presented. The system, which resembles the characteristics of a two-dimensional anisotropic network, is analyzed in terms of the current percolation theory. Parameters such as the percolation threshold and the critical exponents are calculated for the perpendicular and the parallel orientation. Within the limits of the experiments the values of $t_{\|}$and $t_{\perp}$ are estimated to be 1.15 and 1.46 , respectively.
\end{abstract} [S0163-1829(97)03304-3]

\section{INTRODUCTION}

In situ transport measurements during film growth usually show three different stages: an exponential stage due to an activated mechanism, a percolative stage, and an Ohmic stage with additional surface and/or grain boundary scattering. ${ }^{1-4}$ Close to the percolation threshold, the resistance depends on the thickness as

$$
R=R_{0}\left(d-d_{c}\right)^{-t} \quad\left(d>d_{c}\right),
$$

where $d_{c}$ is the critical thickness, $t$ the conductivity critical exponent, and $R_{0}$ is assumed to be constant. Equation (1) is valid if the substrate coverage $\theta$ scales linearly with the film thickness. Experimentally obtained values for the critical exponent $t$ (1.27-1.36) (Refs. 1-4) agree well with the theoretical values (1.29-1.303) for isotropic two-dimensional systems. ${ }^{6-8}$ Conduction in metal films can start as early as $0.2 \mathrm{~nm}$, but even values up to $18 \mathrm{~nm}$ have been reported. ${ }^{1-5,9,10}$ The nominal thickness of the onset $d_{0}$ depends strongly on the substrate-deposit interaction and the conditions of the evaporation. A more convenient control parameter is the substrate coverage rather than the nominal thickness. In the case of isotropic two-dimensional systems it is expected that the critical surface coverage $\theta_{c}$ has values of about $50-67 \%$.

The application of a voltage during film growth usually affects the film morphology. This is in particular the case close to the percolation threshold where high current densities in narrow necks of the percolating path lead to dissipation of thermal energy. Breakdown occurs whenever a microbridge reaches the melting temperature of the film. The resistance of the film may either increase when microbridges are burned or it may decrease when the microchannel be- comes wider. It has been reported that the breakdown current $I_{c}$ scales as $I_{c} \propto B^{-x} . B=V_{3 f} / I^{3}$ is the normalized third harmonic generated by the film, and it is associated with local Joule heating. ${ }^{11,12}$

In studies of anisotropic three-dimensional systems, e.g., polymer matrices with conducting fibers as a filler, it has been found that the resistance becomes anisotropic upon deviation from the threshold. The threshold itself, e.g., the critical volume fraction $\omega_{c}$, is isotropic $\left(\omega_{c \|}=\omega_{c \perp}\right)$, but its value depends strongly on the fiber orientation, the fiber length distribution and on the aspect ratio. ${ }^{13,14}$ It has been concluded from both facts (anisotropic resistance, isotropic onset) that the conductivity exponent $t$ becomes anisotropic upon deviation from the threshold. Computer simulations have shown that this behavior also holds for anisotropic twodimensional systems, but that the critical coverage $\theta_{c}$ depends far less on the aspect ratio than the critical volume fraction. ${ }^{15}$ These results were partially confirmed by a study of anisotropic conducting paper, and values of 1.1-1.2 for $t_{\|}$ and 1.5 for $t_{\perp}$ were reported. ${ }^{16}$

In a previous study tellurium thin films evaporated at room temperature onto oriented poly(butene-1) were investigated by means of transmission electron microscopy and in situ transport measurements. ${ }^{17}$ The morphology of the films resembled that of an isotropic two-dimensional network formed by semispherical islands. Conduction started at a rather large value of $4.7 \mathrm{~nm}$. However, this value agrees well with the experience that tellurium shows a comparatively low surface coverage onto poly(butene-1) substrates. In particular this is the case at elevated substrate temperatures $(323-403 \mathrm{~K})$ and at low evaporation rates $\left(<0.1 \mathrm{~nm} \mathrm{~s}^{-1}\right){ }^{18}$ After deposition, the in situ monitored current increased and leveled off two times, but on different time scales. Both increases were explained by the mutual coalescence of islands 
and the transition of initially amorphous islands to the crystalline state, respectively. The increases took place at a fixed nominal thickness, thus we were not able to analyze the data in terms of Eq. (1).

In the present study we attempt to analyze in situ transport measurements in an anisotropic two-dimensional network in terms of percolation theory. In order to obtain an anisotropic system, the evaporation of tellurium was performed at elevated temperature $\left(T_{s}=350 \mathrm{~K}\right)$. Under these conditions tellurium grows as a fiberlike crystallite with an preferred alignment parallel to the axis of orientation of the poly(butene-1) substrate ${ }^{18}$ furthermore it nucleates in a crystalline form. ${ }^{19,20}$ Hence we did not expect that structural changes, as reported for evaporation at room temperature, would complicate the analysis.

\section{EXPERIMENT}

Highly oriented thin films of poly(butene-1) $\left(M_{w}=1 \times 10^{6}\right)$ were obtained by the spinning method. ${ }^{21}$ The polymer films were spun onto glass supports of size $16 \times 10$ $\mathrm{mm}^{2}$ with either the preferred orientation parallel or perpendicular to the axis of the glass supports. Gold was sputtered onto the polymer surface to a thickness of approximately 100 $\mathrm{nm}$ in order to perform Ohmic contacts with the tellurium, ${ }^{22}$ leaving a free surface between the parallel gold contacts of $9 \times 9 \mathrm{~mm}^{2}$. The supports were mounted into a vacuum chamber $\left(p<3 \times 10^{-4} \mathrm{~Pa}\right)$ and subsequently evaporated with tellurium $(99.999 \%)$ at $350 \mathrm{~K}$. The nominal thickness was monitored by a quartz crystal. All samples were evaporated at the same rate of $0.075 \mathrm{~nm} \mathrm{~s}^{-1}$. A voltage of $5 \mathrm{~V}$ was applied to the gold contacts, and the current was monitored by an picoamperemeter (KEITHLEY 485) during and after evaporation. Transmission electron microscopy (TEM) were performed in a JEOL2000FX microscope operated at 200 $\mathrm{kV}$. For this purpose the thin films were cut into pieces of size $3 \times 3 \mathrm{~mm}^{2}$, and were mounted onto copper grids.

\section{MORPHOLOGY OF THE TELLURIUM THIN FILMS}

Representative morphologies for two different nominal thicknesses are presented in Figs. 1 and 2. As expected, the deposit grows as a fiberlike crystallite with preferred orientation parallel to the substrate orientation. The fiber orientation distributions of two samples each evaporated with applied voltage but with different orientation are compared to that of a sample evaporated without applied voltage. No significant differences between the distributions are found (Fig. $3)$. Moreover, the nucleation densities of the three samples are of the same order of magnitude $\left[\approx(2-4) \times 10^{9} \mathrm{~cm}^{-2}\right]$, and the fiber lengths show the same broad range $(0.1-1.5 \mu \mathrm{m})$.

As expected, the substrate coverage was found to be rather low. We estimate that it does not exceed $30 \%$ and $70 \%$ at nominal thicknesses of 27 and $60 \mathrm{~nm}$, respectively (see Figs. 1 and 2). The low surface coverage is most likely due to a weak interaction between the tellurium admolecules $\left(\mathrm{Te}_{2}\right)$ and the poly(butene-1) substrate. We suggest that a considerable amount of admolecules reevaporate and that high surface diffusion leads to a competitive growth. Both phenomena lead to a rather open network of fibers. It was

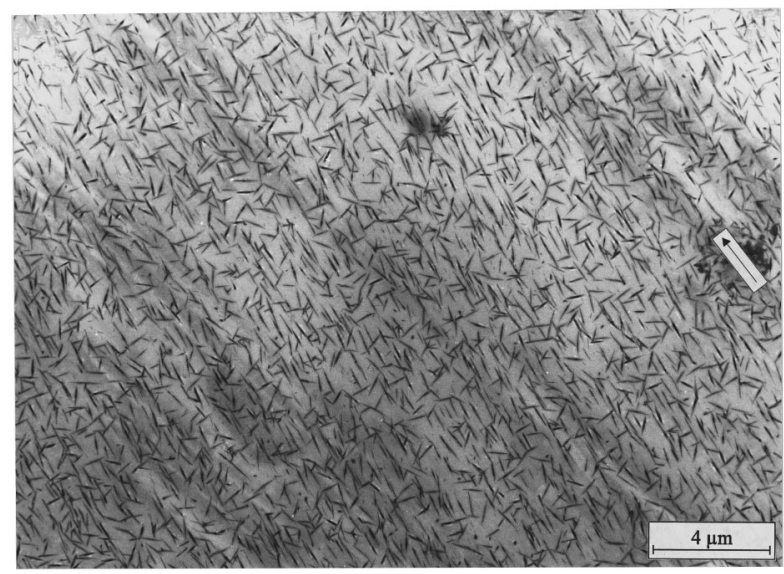

FIG. 1. Micrograph of a 27-nm thin layer of tellurium deposited onto oriented poly(butene-1) (at $T_{s}=350 \mathrm{~K}$ and $R=0.075 \mathrm{~nm} / \mathrm{s}$ ). The arrow indicates the orientation of the poly(butene-1) substrate, and the voltage was applied parallel to the substrate orientation.

checked if any structural changes do occur under the conditions applied in the present work. Contrary to the results obtained at room temperature, the current always decreases when the deposition is stopped close to the onset $d_{0}$ (Fig. 4).

In order to check if the applied voltage has any influence on the morphology of the tellurium films over 30 samples were carefully examined by means of TEM. In none of the samples are traces of a local melting process found, not even in the case of samples with a nominal thickness very close to the onset $d_{0}$. We might speculate that tellurium films are less sensitive to breakdown processes than metal thin films. The conductivity of tellurium is some orders of magnitude lower than those of metals, e.g., the lowest film resistance measured in our experiments is of the order of $10^{5} \Omega$, whereas metal thin films showed resistances of about $10^{2} \Omega .^{1-3}$ Thus we might expect that the current densities are about three orders of magnitude lower than in metal films. Some evidence that the applied voltage might influence the film resistance is discussed below (see Sec. IV).

In summary, it is found that the morphology of the tellu-

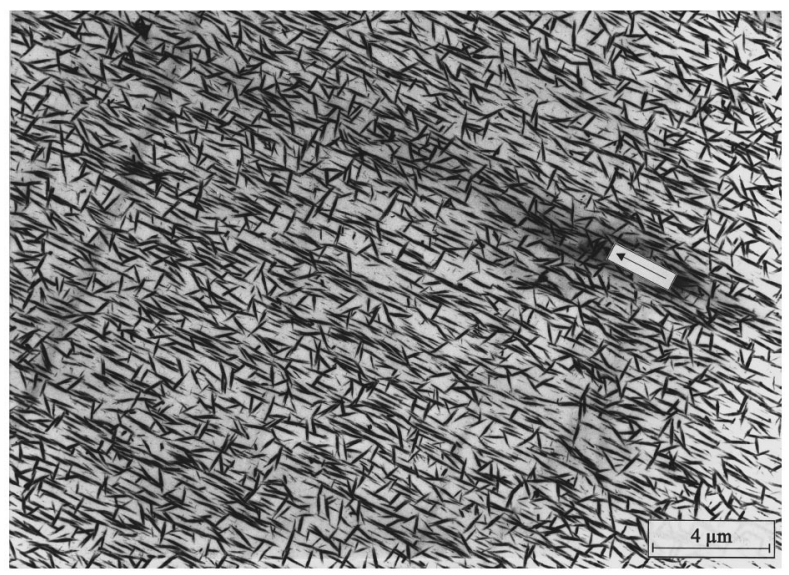

FIG. 2. Micrograph of a 50-nm thin layer of tellurium deposited onto oriented poly(butene-1) (at $T_{s}=350 \mathrm{~K}$ and $R=0.075 \mathrm{~nm} / \mathrm{s}$ ). The arrow indicates the orientation of the poly(butene-1) substrate, and the voltage was applied perpendicular to the substrate orientation. 


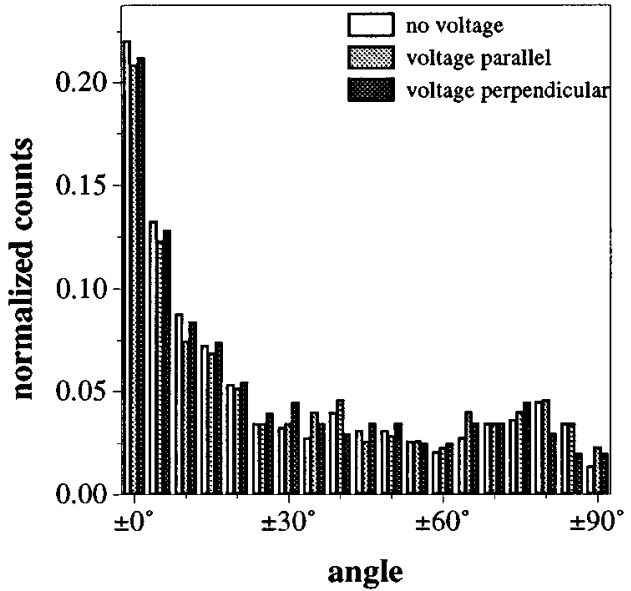

FIG. 3. Orientation distributions of tellurium fibers evaporated onto poly(butene-1). Distributions as obtained with and with no voltage applied during growth are compared to each other.

rium thin films resembles that of an anisotropic twodimensional network formed by fiberlike crystallites. The applied voltage does not affect important morphological parameters like the nucleation density and the fiber orientation distribution. In particular, the fiber orientation is found to be independent of the orientation of the substrate with respect to the applied field. In other words, both sample orientations show the same degree of anisotropy. Small local differences in these parameters are most likely the result of inhomogeneties of the substrate.

\section{ANALYSIS OF TRANSPORT DATA}

Figure 5 shows the experimental data for one sample of each orientation. The conduction onset $d_{0}$ shows a rather large scattering with a mean averages of $17.9 \pm 4.5 \mathrm{~nm}(24$ samples) and 15.4 $\pm 3.4 \mathrm{~nm}$ (ten samples) for parallel and perpendicular orientations, respectively. Scattering of the conduction onset has also been reported for metal thin films

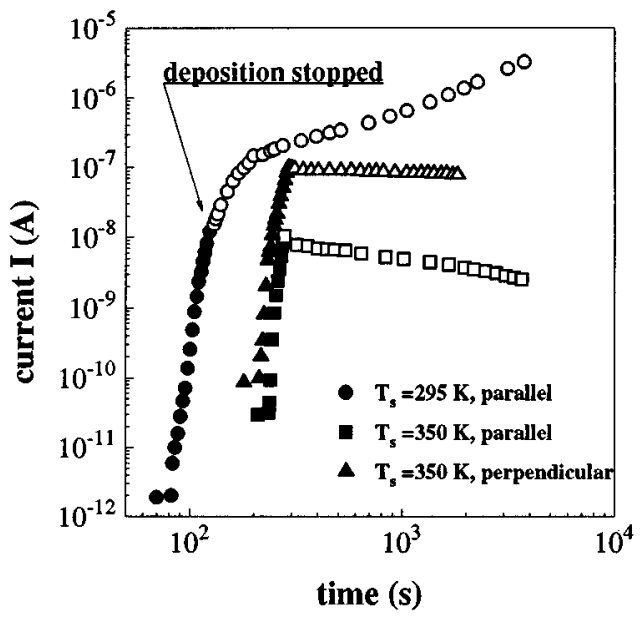

FIG. 4. Comparison of the current-time dependency for samples evaporated at $T_{s}=350 \mathrm{~K}$ and a sample evaporated at $T_{s}=295 \mathrm{~K}$. Filled symbols represent the data which were taken during the evaporation.

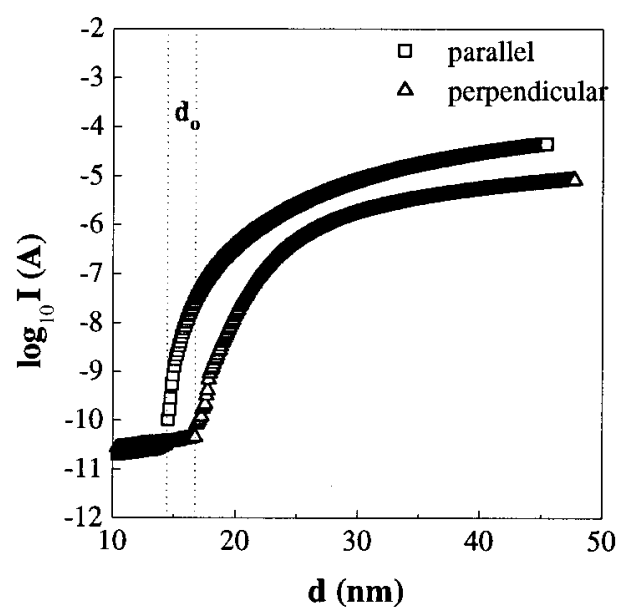

FIG. 5. Current vs thickness curves as obtained during evaporation of tellurium onto oriented poly(butene-1) at $350 \mathrm{~K}$ (deposition rate $0.075 \mathrm{~nm} / \mathrm{s}$ ). Data are shown for both parallel and perpendicular directions.

evaporated at room temperature, ${ }^{1,2}$ and a better reproducibility of the experiment was found only at lower substrate temperatures. ${ }^{1}$ We suggest that the comparatively weak interaction between the polymer substrate and the tellurium leads to a situation in which nucleation is even more sensitive to substrate inhomogeneties. However, we believe that the conduction onset is isotropic $\left(d_{0 \|}=d_{0 \perp}\right)$ with a common mean average of $17.2 \pm 4.3 \mathrm{~nm}$. Despite the different onset values, the data show very similar behaviors. Figure 6 depicts the resistance curves of various parallel oriented samples. Each curve is shifted to the mean average of the onset. They show spreading only close to the onset, and it seems likely that this behavior is caused by local breakdown processes. However, the data coincide well at a later stage of growth, so it does not seem that breakdown occurs at this stage. A similar behavior is observed for the resistance measured in the perpendicular direction. Therefore, we find it justified to represent the data by their mean averages (Fig. 7), and to carry out further analysis on the mean averages of the

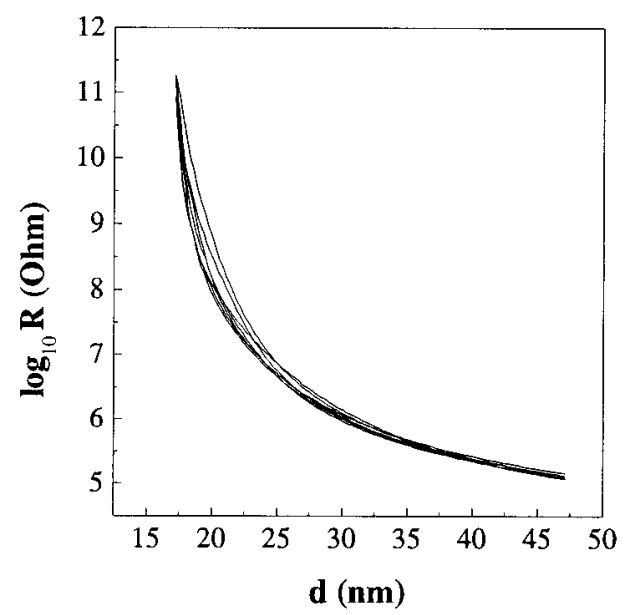

FIG. 6. A set of the resistance curves for the parallel direction. The curves are shifted from their specific onset $d_{0}$ toward the mean average value $\bar{d}_{0}$. 


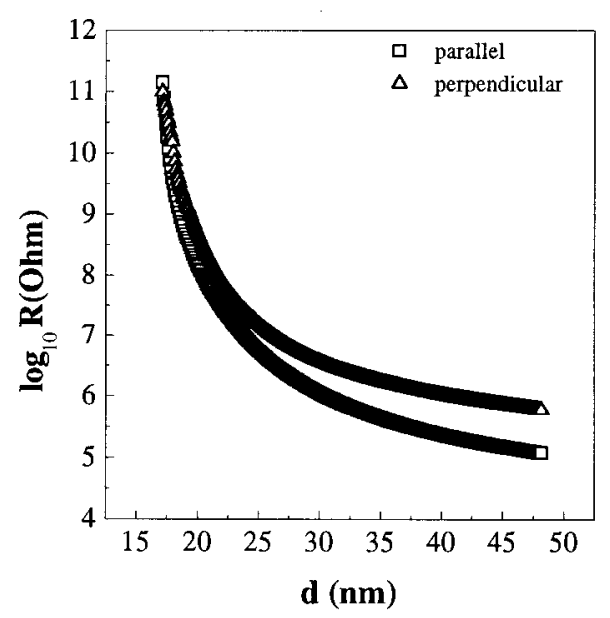

FIG. 7. The calculated mean average of the resistance for samples measured in both parallel and perpendicular directions.

resistance for both orientations. Moreover, a shift of the data along the axis of thickness will not affect the analysis of the critical exponent, but will affect the values of $d_{c}$.

In order to separate the exponential stage from the percolative one, we apply the method of sliding least-square fit. ${ }^{3}$ The method consists of dividing the data into intervals of a given length $( \pm 1-3 \mathrm{~mm})$. By fitting relation (1) to the intervals values of $d_{c}$ and $t$ corresponding to the lowest value of $\chi^{2}$ obtained. The quality of the fits improves from a certain thickness: $\approx 36$ and $\approx 40 \mathrm{~nm}$ for the parallel and perpendicular cases, respectively. When approaching these thicknesses from below, the values of $\chi^{2}$ show a steep decrease. The steep decrease is followed by a region of slightly decreasing values of $\chi^{2}$. In the case of metal thin films, a broad minimum for $\chi^{2}$ was reported, and it was assumed that percolation theory did hold in the range of the minimum, bounded at lower thicknesses by the exponential stage and at larger thicknesses by the Ohmic stage. ${ }^{3}$ Fits of the relation $R d=a_{0}+a_{1} / d+a_{3} / d^{3}$ to our data do not show any satisfactory agreement. Hence we conclude that Ohmic behavior does not apply to the data.

The values of $t$ and $d_{c}$ differ somewhat for different interval length. However, it is possible to calculate the mean average for both, $t$ and $d_{c}$. Figure 8 depicts the obtained values for the critical exponents for both sample orientations. It can be seen that both critical exponents $t_{\|}$and $t_{\perp}$ decrease over the data range where percolation theory holds. Both seem to level off at the largest thicknesses. We will discuss this behavior in terms of a model which accounts for a growth dependency of the local resistance $r$ of the microbridges (or bonds) in the infinite cluster, or, in other words, with a growth-dependent distribution of local resistance (or bond strength) (see Sec. V).

\section{DISCUSSION}

It has been shown that broad conductivity distributions of the form $f(\sigma) \propto \sigma^{-w}$ could lead to a change of the value of $t$ when $w$ is larger than some critical value $w_{c} \cdot{ }^{23,24}$ The results obtained by extensive simulations of isotropic twodimensional random lattices at the threshold $p_{c}$ imply that $t$ equals the universal value of $\approx 1.3$ for $w<w_{c}$. For $w>w_{c}, t$

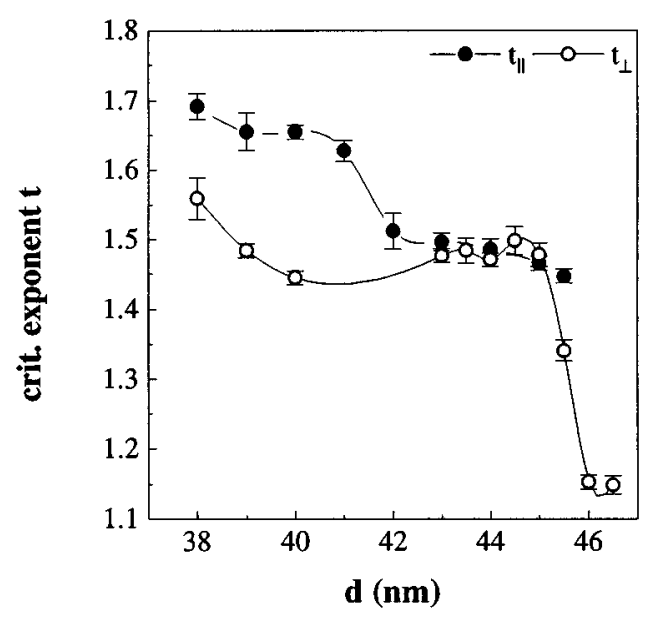

FIG. 8. Behavior of the critical exponents $t_{\|}$and $t_{\perp}$ vs the thickness of the samples. Values are obtained by fitting percolation theory to the mean average of resistance.

has a value given by the estimate $t / \nu=(\nu(1-w))^{-1} \cdot{ }^{23,24} \mathrm{We}$ will discuss why we expect a sufficiently broad distribution of local resistance in our samples, how this distribution might change throughout the growth process, and finally how the effects of the distribution superimpose on the sample anisotropy.

A microbridge is formed whenever the tip of a fiber reaches the lateral surface of another fiber. At this stage of microbridge formation we expect the maximum local resistance, which we denote as $r_{\max }$. Growth leads to a filling up of the narrow microbridge to the full diameter of the fiber; thereafter improvement of the microbridge can only be reached through a thickening of the whole fiber. We expect that the filling up of the microbridge is fast in comparison to the thickening of the whole fiber. Transport measurements of tellurium thin films have been explained by an activated conduction mechanism due to barriers located at grain boundaries (in the temperature range $250-450 \mathrm{~K}$, tellurium evaporated between 295 and $413 \mathrm{~K}$ ). ${ }^{25-28}$ Therefore we expect an exponential decrease of the microbridge resistance during the filling-up process. Whenever the microbridge has been completed, the resistance changes only slightly. We write $r_{\min }$ in order to account for this situation, and note that the resistance distribution spans a broad range due to the exponential decrease from $r_{\max }$ to $r_{\min }$.

In the nodes-links-blobs picture of the infinite cluster, ${ }^{29}$ the total resistance is bounded from below by the narrowest singly connected link, e.g., in our case by the last microbridge formed when the film achieved continuity. Hence the distribution of local resistance includes all values from $r_{\max }$ to $r_{\text {min }}$. Upon deviation from $p_{c}$ the microbridges which were present when the film achieved continuity will approach values close to $r_{\text {min }}$, whereas newly added microbridges will always start with a value close to $r_{\max } \gg r_{\min }$. In other words, the current will be carried mainly by a network of microbridges with values of the order of $r_{\min }$, and a newly added microbridge will contribute significantly to the total resistance $R$ when its local resistance approaches $r_{\text {min }}$. Thus the local resistance distribution of the current-carrying network is narrow. In summary, at $p_{c}$ we expect that the critical exponent takes a value $t(w)$, whereas upon deviation from 
$p_{c}$ it will have its universal value $t$.

The effects of a changing distribution are superimposed on to those of anisotropy. At $p_{c}$ we expect the same critical exponent $t(w)$ for both orientations of the sample $\left[t(w)=t_{\|}(w)=t_{\perp}(w)\right]$. For $p>p_{c}$ and sufficiently large, such that $w<w_{c}$, the local resistance distribution will not affect the values of the anisotropic exponents, and we have $t_{\perp}<t<t_{\|}$. As a consequence, both critical exponents should decrease, but the exponent related to the perpendicular direction should decrease even more strongly [from the value $t(w)$ to $\left.t_{\perp}<t_{\|}\right]$. We find qualitative agreement between the behavior of the experimentally obtained critical exponents and our assumptions. We believe that the final values for $t_{\|}$ and $t_{\perp}$ represent the anisotropy of the systems without superposition of the distribution effects. The mean averages are found to be $1.464 \pm 0.018$ and $1.150 \pm 0.010$, respectively. Both values agree well with those reported in Ref. 16. The values of $d_{c}$ which correspond to those values of the exponents are not distinguishable within the error of our experiment (26.3 and $27.4 \mathrm{~nm}$, respectively). Moreover, both thickness values are close to those which one would expect for the threshold by inspection of the micrographs (see Fig. 1). A plot of $\log _{10} R$ versus $\log _{10}\left(d-d_{c}\right)$ with $d_{c}=27 \mathrm{~nm}$ shows a linear behavior for the last data points (Fig. 9). The plot includes theoretical curves which correspond to the estimations of $t_{\|}$and $t_{\perp}$ given above. Despite the $a d$ hoc analysis of the data, a rather good agreement between theory and experimental data is found.

\section{CONCLUSIONS}

The growth of tellurium crystals at $350 \mathrm{~K}$ onto oriented substrates of poly(butene-1) leads to a rather open network of fiberlike crystallite. The morphology of the tellurium film resembles that of an anisotropic two-dimensional network. The anisotropy of the films does not depend on the applied voltage. Scattering of the conduction onset $d_{0}$ is most likely due to inhomogeneties of the substrate. Close to the onset it seems likely that breakdown processes affect the resistance.

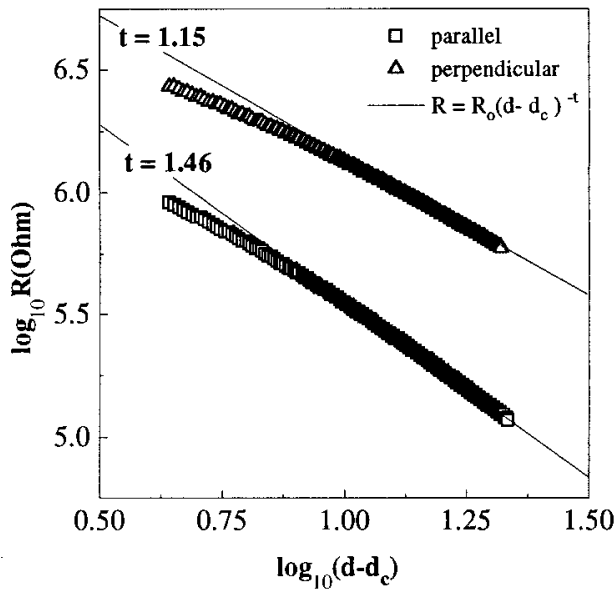

FIG. 9. The $\log _{10} R$ vs $\log _{10}\left(d-d_{c}\right)$ dependency of the mean average curves for both parallel and perpendicular directions. The straight lines represent the theoretical curves as obtained by percolation theory.

Nonetheless, the corrections applied to each particular sample allow us to analyze the system in terms of percolation theory. Our results confirm that the percolation threshold is isotropic $\left(d_{c \|}=d_{c \perp}\right)$. We explain the decrease of the critical exponents $t_{\|}$and $t_{\perp}$ qualitatively by a growth-dependent distribution of bond resistance. Upon deviation from the threshold we expect that the bond resistance distribution becomes narrow, such that $t_{\|}$and $t_{\perp}$ have values which are only affected by the anisotropy. The calculated values of 1.15 and 1.46 agree well with other experimentally obtained values (1.1-1.2 and 1.5 for $t_{\|}$and $t_{\perp}$, respectively).

\section{ACKNOWLEDGMENTS}

Financial support by the Volkswagen Foundation and Comision Interministrial de Ciencia y Tecnologia (Grant No. MAT94-0825) are gratefully acknowledged.
${ }^{1}$ M. Octavio, G. Gutierrez, and J. Aponte, Phys. Rev. B 36, 2461 (1987).

${ }^{2}$ J. A. J. Lourens, S. Arajs, H. F. Helbig, El-Sayed A. Mehana, and L. Cheriet, Phys. Rev. B 37, 5423 (1988).

${ }^{3}$ L. Cheriet, H. H. Helbig, and S. Arajs, Phys. Rev. B 39, 9828 (1989).

${ }^{4}$ V. Korobov, M. Leibovitch, and Y. Shapira, Appl. Phys. Lett. 65, 2290 (1994).

${ }^{5}$ A. Hoareau, J. X. Hu, P. Jensen, P. Melinon, M. Treilleux and B. Cabaud, Thin Solid Films 209, 161 (1992).

${ }^{6}$ J. G. Zabolitsky, Phys. Rev. B 30, 4077 (1984).

${ }^{7}$ J. M. Normand, H. J. Herrmann, and M. Hajjar, J. Stat. Phys. 52, 441 (1988).

${ }^{8}$ D. J. Frank and C. J. Lobb, Phys. Rev. B 37, 302 (1988).

${ }^{9}$ R. S. Markiewicz and L. A. Harris, Phys. Rev. Lett. 46, 1149 (1981).

${ }^{10}$ M. J. Burns and P. M. Chaikin, Phys. Rev. B 27, 5924 (1983).

${ }^{11}$ Y. Yagil, G. Deutscher, and D. J. Bergman, Phys. Rev. Lett. 69, 1423 (1992).
${ }^{12}$ Y. Yagil and G. Deutscher, Phys. Rev. B 46, 16115 (1992)

${ }^{13}$ A. Celzard, E. McRae, C. Deleuze, M. Dufort, G. Furdin, and J. F. Mareché, Phys. Rev. B 53, 6209 (1996).

${ }^{14}$ I. Balberg, N. Binenbaum, and N. Wagner, Phys. Rev. Lett. 52, 1465 (1984).

${ }^{15}$ I. Balberg and N. Binenbaum, Phys. Rev. B 28, 3799 (1983).

${ }^{16}$ K. S. Mendelson and F. G. Karioris, J. Phys. C 13, 6197 (1980).

${ }^{17}$ T. Hoffmann, B. Fiedler, T. Kluck, J. Petermann and J. MartínezSalazar, Thin Solid Films 245, 272 (1994).

${ }^{18}$ J. Petermann and T. Hoffmann, in Advanced Materials and Processes, edited by H. F. Exner and V. Schumacher (DGM Informationsgesellschaft mbH, Oberursel, 1990), Vol. 2, p. 881.

${ }^{19}$ M. Janda and A. Kubový, Phys. Status Solidi A 35, 391 (1976).

${ }^{20}$ K. Okuyama, M. Chiba, and Y. Kumagai, Jpn. J. Appl. Phys. 18, 507 (1979).

${ }^{21}$ J. Petermann and R. M. Gohil, J. Mater. Sci. 14, 2260 (1976).

${ }^{22}$ R. Swan, A. K. Ray, and C. A. Hogarth, Phys. Status Solidi A 127, 555 (1991). 
${ }^{23}$ M. Octavio and C. J. Lobb, Phys. Rev. B 43, 8233 (1991).

${ }^{24}$ D. Stauffer and A. Aharony, Introduction to Percolation Theory revised edition (Taylor and Francis, London, 1995), Vol. 2; see Chap. 5 and references herein.

${ }^{25}$ M. J. Capers and M. White, Thin Solid Films 15, 5 (1973).
${ }^{26}$ A. M. Phahle, Thin Solid Films 41, 235 (1977).

${ }^{27}$ A. Kubový and M. Janda, Phys. Status Solidi A 40, 225 (1977).

${ }^{28}$ A. Mansingh and A. K. Garg, Thin Solid Films 131, 41 (1985).

${ }^{29}$ H. E. Stanley, J. Phys. A 10, L211 (1977). 